\title{
Short-range Ferromagnetism in Alloy Ribbons of Fe-Cr-Si-Nb-(Ag, Cu)
}

\author{
Thanh, P. Q.; Hoa, N. Q.; Chau, N.; Huu, C. X.; Ngo, Duc-The; Phan, T. L.
}

Published in:

Journal of the Korean Physical Society

Link to article, DOI:

$10.3938 / j k p s .64 .1016$

Publication date:

2014

Document Version

Publisher's PDF, also known as Version of record

Link back to DTU Orbit

Citation (APA):

Thanh, P. Q., Hoa, N. Q., Chau, N., Huu, C. X., Ngo, D-T., \& Phan, T. L. (2014). Short-range Ferromagnetism in Alloy Ribbons of Fe-Cr-Si-Nb-(Ag, Cu). Journal of the Korean Physical Society, 64(7), 1016-1021.

https://doi.org/10.3938/jkps.64.1016

\section{General rights}

Copyright and moral rights for the publications made accessible in the public portal are retained by the authors and/or other copyright owners and it is a condition of accessing publications that users recognise and abide by the legal requirements associated with these rights.

- Users may download and print one copy of any publication from the public portal for the purpose of private study or research.

- You may not further distribute the material or use it for any profit-making activity or commercial gain

- You may freely distribute the URL identifying the publication in the public portal 


\title{
Short-range Ferromagnetism in Alloy Ribbons of Fe-Cr-Si-Nb-( $\mathrm{Ag}, \mathrm{Cu})$
}

\author{
P. Q. THANH, N. Q. HOA and N. CHAU \\ Faculty of Physics, Hanoi University of Science, Vietnam National University, Hanoi, Vietnam \\ C. X. HUU \\ Department of Electronics and Communication Engineering, \\ Danang University of Technology, Danang, Vietnam \\ D.-T. NGO \\ Department of Micro- and Nanotechnology, and Department of Energy and Storage, \\ Technical University of Denmark, Kgs. Lyngby 2800, Denmark \\ T. L. PHAN* \\ Department of Physics, Chungbuk National University, Cheongju 361-763, Korea
}

(Received 19 December 2013, in final form 13 January 2014)

\begin{abstract}
We have studied the magnetic properties of two amorphous alloy ribbons $\mathrm{Fe}_{72} \mathrm{Cr}_{6} \mathrm{Si}_{4} \mathrm{Nb}_{5} \mathrm{~B}_{12} \mathrm{Ag}_{1}$ (FCSNB-Ag) and $\mathrm{Fe}_{72} \mathrm{Cr}_{6} \mathrm{Si}_{4} \mathrm{Nb}_{5} \mathrm{~B}_{12} \mathrm{Cu}_{1}$ (FCSNB-Cu), prepared by using a melt-spinning technique. Magnetization $(M)$ measurements for various temperatures $(T)$ and magnetic fields $(H)$ indicate that ferromagnetic-paramagnetic (FM-PM) phase transitions take place in FCSNB-Ag and FCSNB-Cu at Curie temperatures $\left(T_{C}\right)$ of about $308.3 \mathrm{~K}$ and $322.5 \mathrm{~K}$, respectively. Analyses of $M-H$ data at different temperatures in the vicinity of the FM-PM phase transition based on the modified Arrott plot method and scaling hypothesis yielded the exponent values of $\beta=0.369 \pm$ $0.005, \gamma=1.359 \pm 0.005$ and $\delta=4.7 \pm 0.1$ for FCSNB-Ag, and $\beta=0.376 \pm 0.002, \gamma=1.315$ \pm 0.006 and $\delta=4.5 \pm 0.1$ for FCSNB-Cu. Compared with the values from theoretical models, these values are close to those expected for the 3D Heisenberg model, demonstrating the existence of short-range FM order in the amorphous alloy ribbons.
\end{abstract}

PACS numbers: 75.20.En, 75.30.Cr, 75.40.-s

Keywords: Alloy ribbons, Critical behavior, Short-range magnetic order

DOI: $10.3938 /$ jkps.64.1016

\section{INTRODUCTION}

Currently, Fe-, Ni- and Co-based amorphous alloys are still an issue of intensive interest because of their promising applications in devices for conversion of electromagnetic energy into mechanic energy, magnetic refrigerator, sensitive sensors, telecommunications, automotive magnetics, and electronic article surveillance [1-4]. Compared with the conventional alloys $\mathrm{NiFe}, \mathrm{FeCo}$ and $\mathrm{FeSi}$, these amorphous alloys exhibit some rotable properties, such as high corrosion resistance, relatively great saturation magnetization, and soft-magnetic behavior (i.e., small coercivity, leading to low hysteretic losses). Depending on the applications, these properties can be easily modified by changing the dimension and compositions of the amorphous alloys. These amorphous alloys are a promising new class of materials where applications

*E-mail: ptlong2512@yahoo.com; Fax: +82-43-275-6416 are in competition with those of the conventional alloys. Particularly, the addition of $\mathrm{Ti}, \mathrm{Cr}, \mathrm{Nb}, \mathrm{Si}, \mathrm{B}$ and/or $\mathrm{Cu}$ to $\mathrm{Fe}-$ and $\mathrm{Co}-\mathrm{metalloid}$ alloys remarkably improves their corrosion resistance and soft-magnetic behaviors $[2,5-7]$. Here, the combination of chemical elements with different atomic radii stimulates structural disorder (short-range structure) so that the amorphous phase can be easily formed and ferromagnetic-paramagnetic (FM$\mathrm{PM}$ ) phase-transition temperature (the Curie temperature, $T_{C}$ ) can be controlled.

Recently, more and more attention has focused on fabricating and studying amorphous alloy ribbons for magnetic-refrigeration technology, which is based on the magnetocaloric (MC) effect $[2,3,8-10]$. This effect is directly related to the temperature change (or magneticentropy change) under adiabatic conditions of a ferromagnet under an external applied field. The temperature (or magnetic-entropy) change is strongest around the FM-PM phase-transition temperature $T_{C}$, where 
magnetic moments become disordered due to the thermal activation energy [11]. For conventional applications in cooling systems (such as air conditioners, refrigeration and freezers), the $T_{C}$ of amorphous alloys can be controlled in the temperature range of $260 \sim$ $310 \mathrm{~K}$. For example, the $T_{C}$ values of the amorphous alloy ribbons $\mathrm{Fe}_{78} \mathrm{Nb}_{5} \mathrm{Si}_{4} \mathrm{~B}_{12} \mathrm{Cu}_{1}(>450 \mathrm{~K})[8,9]$ and $\mathrm{Fe}_{73.5} \mathrm{Nb}_{3} \mathrm{Si}_{13.5} \mathrm{~B}_{9} \mathrm{Au}_{1}$ (>600 K) [12] can be reduced to lower temperatures by doping $\mathrm{Cr}$ into the $\mathrm{Fe}$ site. Though many works have focused on the physical properties of Cr-doped Fe-Nb-Si-B-Cu-based amorphous alloy ribbons [7-9,13], the magnetic interactions around their FM-PM phase transitions have not been intensively studied. Additionally, no work in reference to the magnetic properties of (Cr, Ag)-codoped Fe-Nb-Si-B alloy ribbons seems to exist. To get more insight into these material systems, we prepared two amorphous alloy ribbons $\mathrm{Fe}_{72} \mathrm{Cr}_{6} \mathrm{Si}_{4} \mathrm{Nb}_{5} \mathrm{~B}_{12} \mathrm{Cu}_{1}$ and $\mathrm{Fe}_{71} \mathrm{Cr}_{7} \mathrm{Si}_{4} \mathrm{Nb}_{5} \mathrm{~B}_{12} \mathrm{Ag}_{1}$, and then studied their magnetic properties based on magnetization $(M)$ versus temperature $(T)$ and magnetic field $(H)$ measurements. Based on the mean-field theory for a second-order magnetic phase transition (SOMT) associated with long-range magnetic interactions [14], we determined the values of the critical exponents $\beta, \gamma$ and $\delta$ associated with the temperature dependences of the saturation magnetization, $M_{s}(T)$, the inverse initial susceptibility, $\chi_{0}^{-1}(T)$, and the critical isotherm $M$, respectively. Compared with the theoretical values, these experimentally-determined values are close to those expected for the 3D Heisenberg model [15], demonstrating the existence of short-range FM order in the amorphous alloy ribbons.

\section{EXPERIMENTAL DETAILS}

Two amorphous ribbons (the widths and thicknesses are of about 2-6 mm and $20 \mu \mathrm{m}$, respectively) with nominal compositions of $\mathrm{Fe}_{71} \mathrm{Cr}_{7} \mathrm{Si}_{4} \mathrm{Nb}_{5} \mathrm{~B}_{12} \mathrm{Ag}_{1}$ (FCSNB-Ag) and $\mathrm{Fe}_{72} \mathrm{Cr}_{6} \mathrm{Si}_{4} \mathrm{Nb}_{5} \mathrm{~B}_{12} \mathrm{Cu}_{1}$ (FCSNB-Cu) were prepared from high-purity elements $(>3 \mathrm{~N})$ as precursors by using the melt-spinning technique. The fabrication was carried out in a vacuum chamber at $10^{-4}$ Torr, and that pressure was maintained by using an Ar gas flow. After preparation, the amorphous phase in the final ribbon products was confirmed by using X-ray diffraction [9]. Magnetization versus temperature and magnetic field measurements were performed on a vibrating sample magnetometer (VSM), where the temperature and the magnetic field could be changed from 100 to about $500 \mathrm{~K}$ and from 0 to $10 \mathrm{kOe}$, respectively. To study the critical behavior of the alloy ribbons, we used Arrott plots $[16,17]$ and the scaling hypothesis [15] to analyze the $M-H-T$ data in the vicinity of the $T_{C}$.

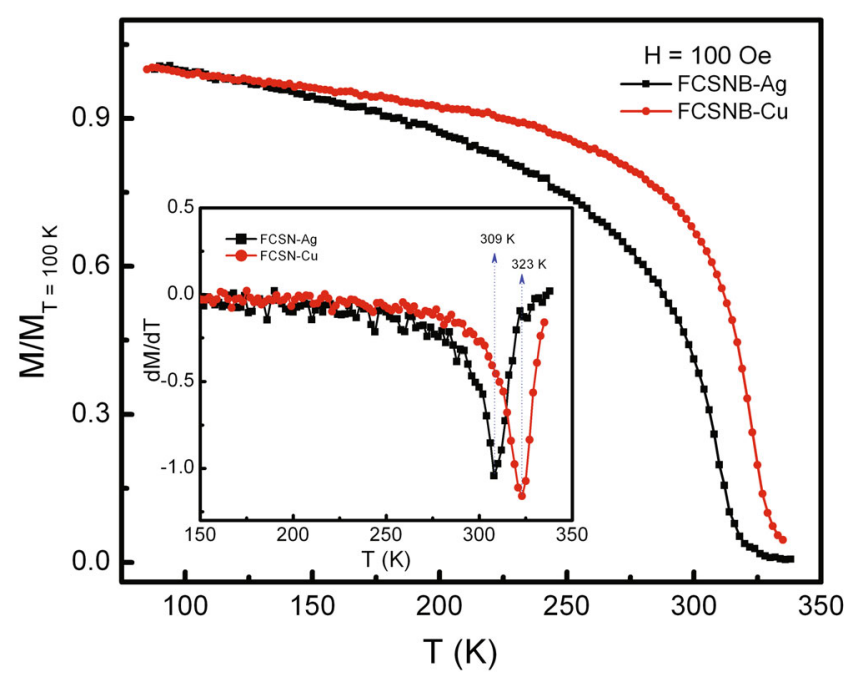

Fig. 1. (Color online) Temperature dependences of magnetization normalized to the value at $100 \mathrm{~K}, M / M_{T}=100$ $\mathrm{K}$, for the amorphous alloy ribbons in the field $H=100$ Oe. The inset shows $\mathrm{d} M / \mathrm{d} T$ versus $T$ curves.

\section{RESULTS AND DISCUSSION}

Figure 1 shows the temperature dependences of the zero-field-cooled magnetization normalized to the value at $100 \mathrm{~K}$ for the amorphous alloy ribbons FCSNB-Ag and FCSNB-Cu in the field $H=100$ Oe. The gradual decrease in the magnetization at low temperatures can be seen to become rapid when the temperature is higher than $290 \mathrm{~K}$, which is due to the FM-PM phase transition taking place at the $T_{C}$. At temperatures above the $T_{C}$, the samples are in the PM state; thus, the magnetization decreases to zero. From the $\mathrm{d} M / \mathrm{d} T$ versus $T$ curves, we obtained the $T_{C}$ values (from the minima of the curves, as shown in the inset of Fig. 1) of FCSNB-Ag and FCSNB-Cu to be about $309 \mathrm{~K}$ and $323 \mathrm{~K}$, respectively. If more attention is given to the variation of the $M(T)$ curves, that variation is quite different, particularly at temperatures above $150 \mathrm{~K}$. While high $M$ values and a narrower FM-PM transition region are observed for FCSNB-Cu, such features are absent from FCSNB$\mathrm{Ag}$. To explain the obtained results, we consider the $\mathrm{Cr}$ content in the alloy ribbons because both $\mathrm{Ag}$ and $\mathrm{Cu}$ in the same amounts are diamagnetic. The $\mathrm{Cr}$ content in FCSNB-Ag (7 at.\%) appears to be higher than that in FCSNB-Cu (6 at.\%). According to previous studies $[8,9]$, the $T_{C}$ and $M$ values of FCSNB-Ag should be smaller than those of FCSNB-Cu. However, such scenarios do not occur in the present case. This proves that the differences in the $T_{C}$ and $M$ values, and in the characteristic phase transition not only depend on Cr-doping content but also on the natures of the $\mathrm{Ag}$ and $\mathrm{Cu}$ elements present in the FCSNB host, where the Fe-related FM phase plays an important role.

To further understand the magnetic properties of FCSNB-Ag and FCSNB-Cu, we recorded magnetic-field 


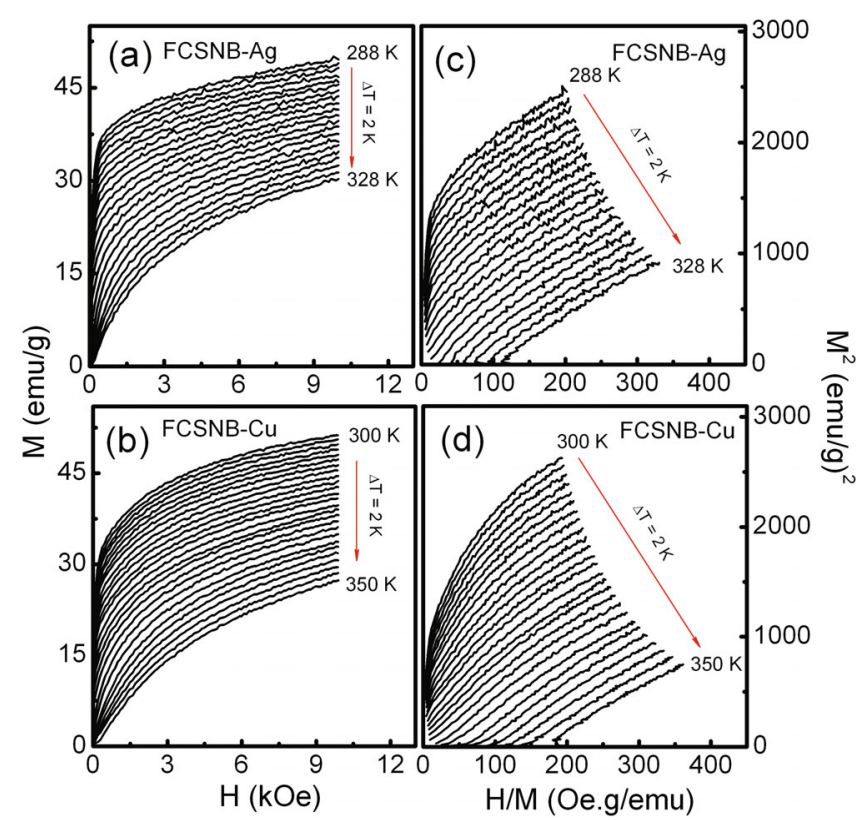

Fig. 2. (Color online) (a, b) $M(H)$ curves and (c, d) Arrott plots of $M^{2}$ versus $H / M$ for amorphous alloy ribbons of FCSNB-Ag and FCSNB-Cu at temperatures around the FM-PM phase transitions.

dependences of the initial magnetization, $M(H)$, at different temperatures and then studied their critical behavior by determining the values of the exponents $\beta, \gamma$ and $\delta$. As shown in Figs. 2(a) and (b), the magnetization gradually decreases, and the nonlinear $M(H)$ curves in the FM region tend to become linear with increasing temperature because the samples go into the PM state. Though the field is increased up to $10 \mathrm{kOe}$, no saturation magnetization occurs. This phenomenon was also observed in $\mathrm{Fe}_{70} \mathrm{Cr}_{8} \mathrm{Si}_{4} \mathrm{Nb}_{5} \mathrm{~B}_{12} \mathrm{Cu}_{1}$ [8] and in other alloy ribbons $[4,18]$, which is a characteristic of ferromagnets without true long-range magnetic order. According to the mean-field theory (MFT) proposed for a ferromagnet exhibiting a SOMT and long-range magnetic interactions [14], the free energy $G_{L}$ can be expanded in even powers of $M$ as follows:

$$
G_{L}=a M^{2}+b M^{4}+\ldots-H M,
$$

where $a$ and $b$ are temperature-dependent parameters. Minimizing $G_{L}$ gives the relation:

$$
H / M=2 a+4 b M^{2} .
$$

This equation indicates that when the magnetic interactions in a ferromagnet obey the MFT, the plots of $M^{2}$ versus $H / M$ around the $T_{C}$ are parallel straight lines. Particularly, the $M^{2}$ versus $H / M$ line at the $T_{C}$ passes through the coordinate origin, as in Arrott plots [16,17]. However, these features are not observed in Figs. 2(c) and (d), which means that magnetic interactions in our amorphous alloys FCSNB-Ag and FCSNB-Cu do not

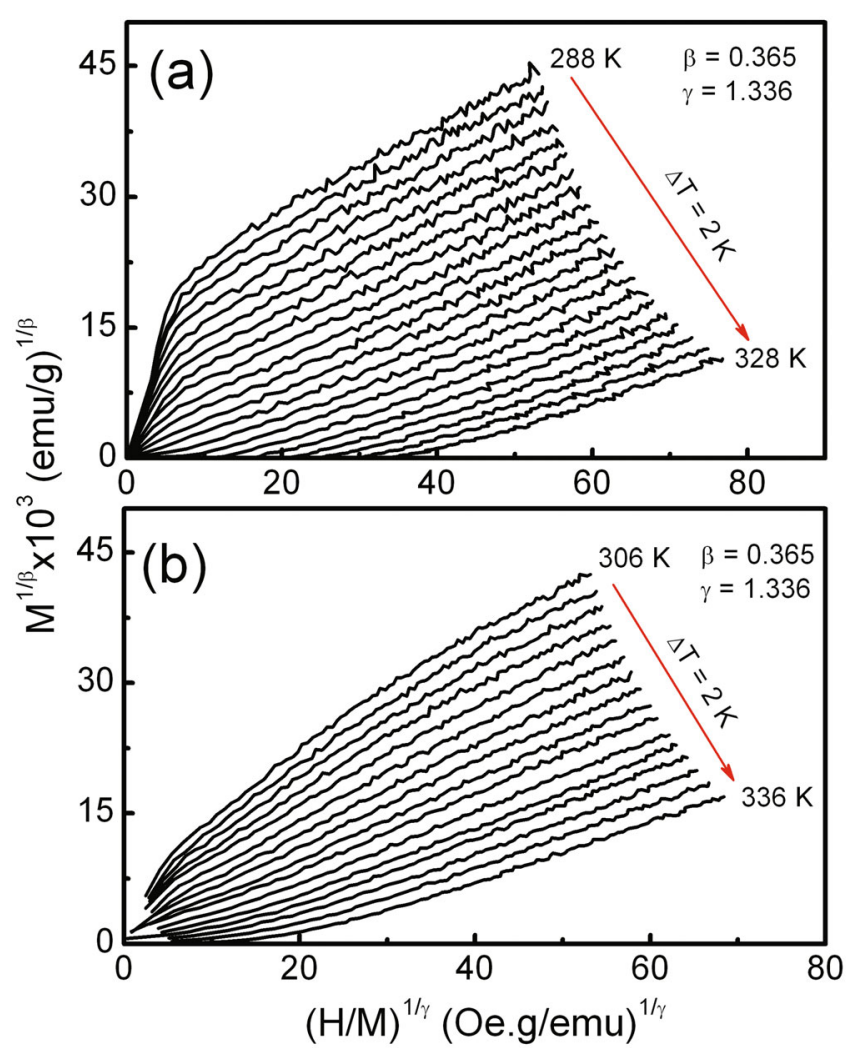

Fig. 3. (Color online) Modified Arrott plots with trial exponents $\beta=0.365$ and $\gamma=1.336$ expected for the $3 \mathrm{D}$ Heisenberg model.

have long-range order. More evidence supporting this conclusion can be gotten from the critical exponents $\beta$, $\gamma$ and $\delta$. Within the framework of the MFT, Eq. (2) for modified Arrott plots (MAP) can be generalized by using the scaling equation of state [17]

$$
(H / M)^{1 / \gamma}=c_{1} \varepsilon+c_{2} M^{1 / \beta},
$$

where $c_{1}$ and $c_{2}$ are temperature-dependent parameters, and $\varepsilon=\left(T-T_{C}\right) / T_{C}$ is the reduced temperature. In this equation, the $\beta, \gamma$ and $\delta$ values can be obtained from the asymptotic relations

$$
\begin{array}{ll}
M_{s}(T)=M_{0}(-\varepsilon)^{\beta}, & \varepsilon<0, \\
\chi_{0}^{-1}(T)=\left(h_{0} / M_{0}\right) \varepsilon^{\gamma}, & \varepsilon>0, \\
M=D H^{1 / \delta}, & \varepsilon=0,
\end{array}
$$

where $M_{0}, h_{0}$, and $D$ are the critical amplitudes. We should notice that if $\beta=0.5$ and $\gamma=1.0$, Eq. (3) returns the form of Eq. (2). However, these $\beta$ and $\gamma$ values are not suitable for describing magnetic interactions taking place in our alloys. Thus, more accurate values are needed. In the present work, we have used the MAP method, starting from the scaling equation of state with trial exponent values of $\beta=0.365$ and $\gamma=1.336$ expected for the 3D Heisenberg model [15]. As mentioned above, correct $\beta$ and $\gamma$ values make the $M(H)$ data falling on a 


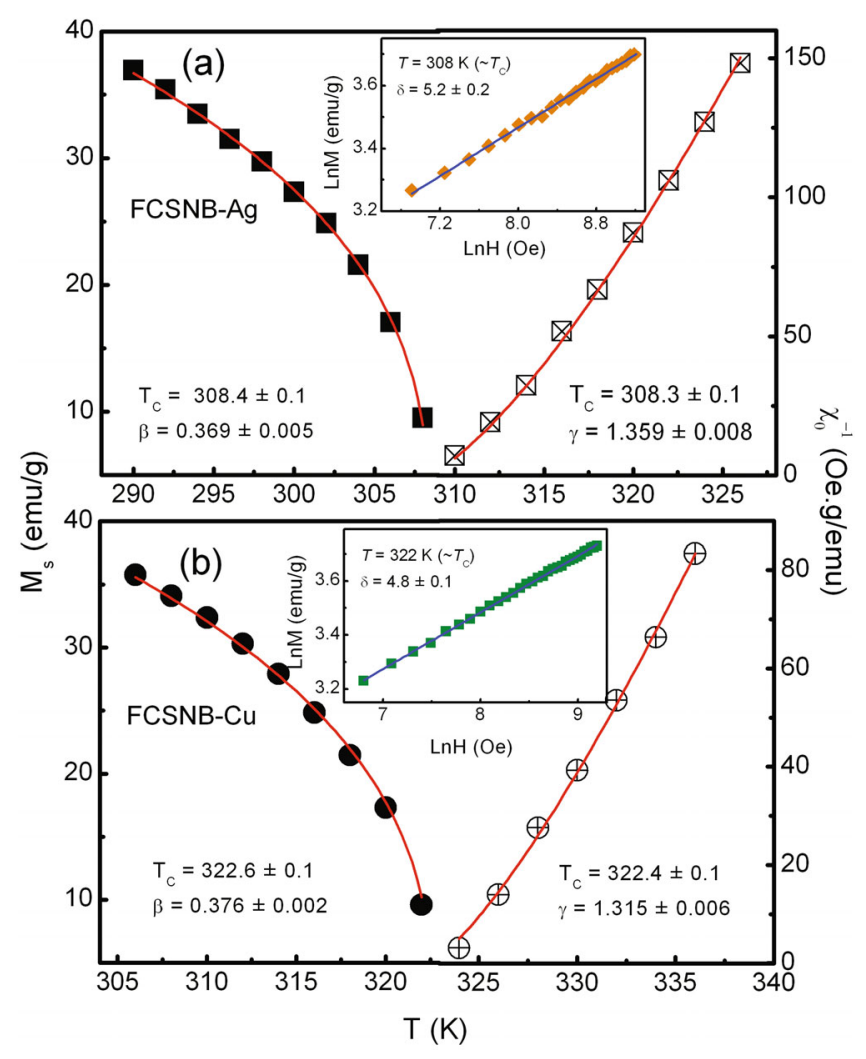

Fig. 4. (Color online) $M_{s}(T)$ and $\chi_{0}^{-1}(T)$ data around $T_{C}$ fitted to Eqs. (4) and (5), respectively, for (a) FCSNB$\mathrm{Ag}$ and (b) FCSNB-Cu. The insets show the isotherms at temperatures close to $T_{C}$ fitted to Eq. (6).

set of parallel straight lines in the performance of $M^{1 / \beta}$ versus $(H / M)^{1 / \gamma}$, and the $M^{1 / \beta}$ versus $(H / M)^{1 / \gamma}$ lines pass through the origin at the $T_{C}$. These features can be clearly seen in Fig. 3 for magnetic fields $H>3 \mathrm{kOe}$, proving that the values of the critical exponents associated with short-range magnetic interactions of the 3D Heisenberg and/or 3D Ising models are more suitable for describing our system.

With the trial exponent values and the MAP shown in Fig. $3, M_{s}(T)$ and $\chi_{0}(T)$ data obtained from the linear extrapolation in the high-field region $(H>3 \mathrm{kOe})$ to the $M^{1 / \beta}$ and $\left(1 / \chi_{0}\right)^{1 / \gamma}$ axes are then fitted to Eqs. (4) and (5), respectively, to achieve better $\beta, \gamma$ and $T_{C}$ values. These new values of $\beta, \gamma$ and $T_{C}$ are continuously used for the next MAP processes until their optimal values are achieved. Notably, during the best fitting process, the $T_{C}$ values determined from the $M(T)$ data (shown in Fig. 1) were used as a reference. The final results are shown in Fig. 4 and Table 1, where the values of the critical parameters are $T_{C} \approx 308.3 \mathrm{~K}, \beta=0.369 \pm 0.005$, $\gamma=1.359 \pm 0.005$ and $\delta=4.7 \pm 0.1$ for FCSNB-Ag and $T_{C} \approx 322.5 \mathrm{~K}, \beta=0.376 \pm 0.002, \gamma=1.315 \pm 0.006$ and $\delta=4.5 \pm 0.1$ for FCSNB-Cu. The $T_{C}$ values obtained in this case can be seen to be very close to those obtained from the $M(T)$ data.

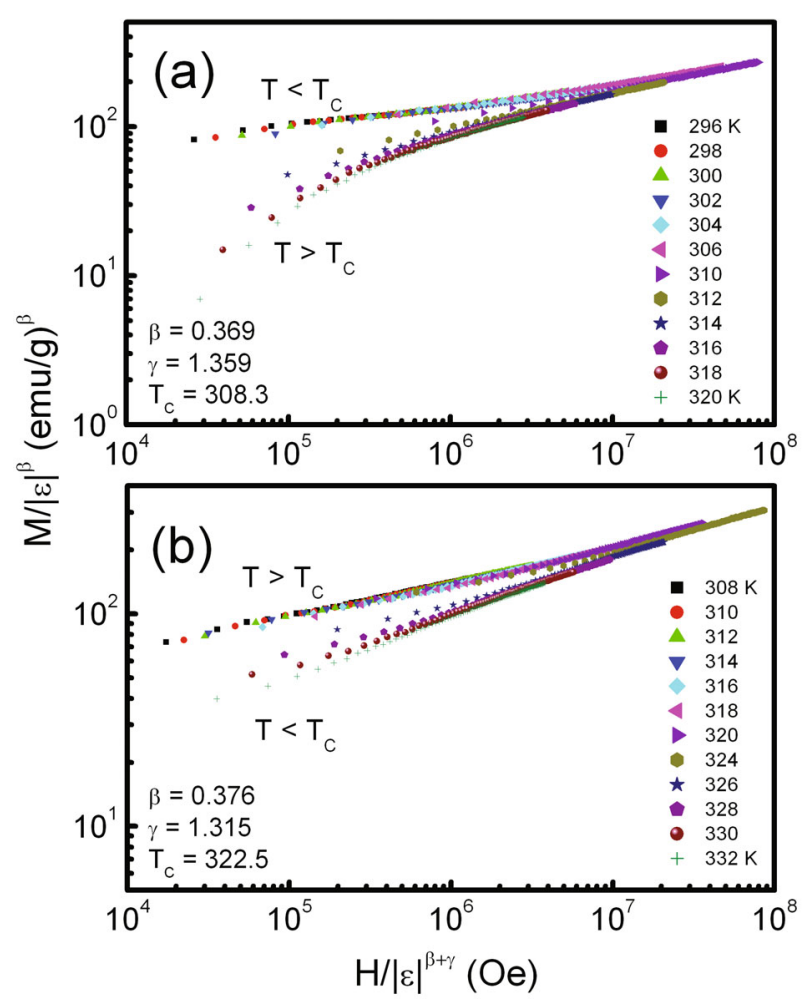

Fig. 5. (Color online) Scaling performance of $M /|\varepsilon|^{\beta}$ versus $H /|\varepsilon|^{\beta+\gamma}$ for the $M-H-T$ data around the $T_{C}$ values of two alloy ribbons: (a) FCSNB-Ag and (b) FCSNB-Cu.

The reliability of the exponent values can be checked by means of the static-scaling hypothesis, which states that $M$ is a function of $\varepsilon$ and $H, M(H, \varepsilon)=|\varepsilon|^{\beta}$ $f_{ \pm}\left(H /|\varepsilon|^{\beta+\gamma}\right)$, where $f_{+}$and $f_{-}$are analytic functions for $T>T_{C}$ and $T<T_{C}$, respectively [15]. This equation implies that, with the determined $\beta$ and $\gamma$ values, all $M(H)$ data points fall onto two universal branches of $f_{-}$and $f_{+}$in $M / \varepsilon^{\beta}$ versus $H / \varepsilon^{\beta+\gamma}$ curves. Clearly, such descriptions are in good agreement with the features of the high-field region shown in Fig. 5, demonstrating the reliability of the $\beta$ and $\gamma$ values determined in our work. It should be noticed that, in the low-field region, due to rearrangement of magnetic domains and the uncertainty in the calculation of demagnetization factor, unexpected errors for critical values can thus be occurred, leading to the scattering of the $M-H$ data points from the universal curves. For the last exponent $\delta$, its values obtained from the Widom relation of $\delta=1+\gamma / \beta$ [15] for FCSNB$\mathrm{Ag}$ and FCSNB-Cu are $4.7 \pm 0.1$ and $4.5 \pm 0.1$, respectively, which are close to those obtained from the fitting the $M(H)$ isotherms at $308 \mathrm{~K}\left(\sim T_{C}\right)$ for FCSNB-Ag and $322.4 \mathrm{~K}\left(\sim T_{C}\right)$ for FCSNB-Cu to Eq. (5), as shown in the insets of Fig. 4.

The comparison of the theoretical and experimental values in Table 1 shows that the $\beta, \gamma$ and $\delta$ values obtained in our work are quite close to those expected from the 3D Heisenberg model with $\beta=0.365, \gamma=1.336$ and $\delta=4.8$. This once again proves the existence of 
Table 1. Values of the critical parameters of FCSNB-Ag and FCSNB-Cu alloy ribbons compared with those of the theoretical models and of some Fe-based amorphous alloys reported previously using the MAP method. Here, $\delta$ values are mainly calculated from the Widom relation $\delta=1+\gamma / \beta$.

\begin{tabular}{|c|c|c|c|c|c|}
\hline Model/Material & $T_{C}(\mathrm{~K})$ & $\beta$ & $\gamma$ & $\delta$ & Ref. \\
\hline Mean-field theory & - & 0.5 & 1.0 & 3.0 & 15 \\
\hline 3D Heisenberg model & - & 0.365 & 1.336 & 4.8 & 15 \\
\hline 3D Ising model & - & 0.325 & 1.241 & 4.82 & 15 \\
\hline $\mathrm{Fe}_{72} \mathrm{Cr}_{6} \mathrm{Si}_{4} \mathrm{Nb}_{5} \mathrm{~B}_{12} \mathrm{Ag}_{1}$ & 308.3 & $0.369 \pm 0.005$ & $1.359 \pm 0.008$ & $4.7 \pm 0.1$ & This work \\
\hline $\mathrm{Fe}_{72} \mathrm{Cr}_{6} \mathrm{Si}_{4} \mathrm{Nb}_{5} \mathrm{~B}_{12} \mathrm{Cu}_{1}$ & 322.5 & $0.376 \pm 0.002$ & $1.315 \pm 0.006$ & $4.5 \pm 0.1$ & This work \\
\hline $\mathrm{Fe}_{70} \mathrm{Cr}_{8} \mathrm{Si}_{4} \mathrm{Nb}_{5} \mathrm{~B}_{12} \mathrm{Cu}_{1}$ & 285 & $0.31 \pm 0.02$ & $1.60 \pm 0.02$ & $6.2 \pm 0.1$ & 8 \\
\hline $\mathrm{Fe}_{76} \mathrm{Cr}_{2.5} \mathrm{~B}_{13.5} \mathrm{Si}_{8}$ & 613 & 0.398 & 1.38 & 4.5 & 19 \\
\hline $\mathrm{Fe}_{75.5} \mathrm{Cr}_{4} \mathrm{~B}_{13} \mathrm{Si}_{7.5}$ & 567 & 0.366 & 1.286 & 4.8 & 19 \\
\hline $\mathrm{Fe}_{73.6} \mathrm{Cr}_{6.5} \mathrm{~B}_{14.6} \mathrm{Si}_{5.3}$ & 430 & 0.358 & 1.365 & 4.8 & 19 \\
\hline $\mathrm{Fe}_{90} \mathrm{Zr}_{10}$ & 227 & 0.345 & 1.395 & 5.0 & 20 \\
\hline $\mathrm{Fe}_{86} \mathrm{Mn}_{4} \mathrm{Zr}_{10}$ & 213 & 0.369 & 1.368 & 4.7 & 20 \\
\hline $\mathrm{Fe}_{84} \mathrm{Mn}_{6} \mathrm{Zr}_{10}$ & 197 & 0.341 & 1.358 & 5.0 & 20 \\
\hline $\mathrm{Fe}_{82} \mathrm{Mn}_{8} \mathrm{Zr}_{10}$ & 185 & 0.365 & 1.387 & 4.8 & 20 \\
\hline $\mathrm{Fe}_{80} \mathrm{Mn}_{10} \mathrm{Zr}_{10}$ & 169 & 0.368 & 1.384 & 4.8 & 20 \\
\hline $\mathrm{Fe}_{78} \mathrm{Mn}_{12} \mathrm{Zr}_{10}$ & 154.3 & 0.359 & 1.378 & 4.8 & 20 \\
\hline$\left(\mathrm{Fe}_{0.74} \mathrm{Cu}_{0.26}\right)_{85} \mathrm{Zr}_{15}$ & 326.5 & 0.52 & 1.01 & 3.15 & 21 \\
\hline $\mathrm{Fe}_{77} \mathrm{Co}_{5.5} \mathrm{Ni}_{5.5} \mathrm{Zr}_{7} \mathrm{~B}_{4} \mathrm{Cu}_{1}$ & 493 & $0.53 \pm 0.03$ & $1.34 \pm 0.04$ & $3.5 \pm 0.4$ & 22 \\
\hline
\end{tabular}

short-range FM order in the amorphous alloy ribbons. Previous studies on some Fe-based amorphous alloy ribbons also revealed the same result $[8,19,20]$. Very few works have found exponent values associated with longrange FM interactions [21,22] (see Table 1). Recently, with simple models of magnetism, Skomski has showed that long-range FM interactions could be established in complicated spin structures, including FM, anti-FM and non-collinear magnetic materials [23]. In fact, the values of the critical exponents depend strongly on the range of the exchange interaction $J(r)$, spins, and the spatial dimensionality. Using the renormalization group approach for an exchange-interaction system, Fisher and co-workers [24] found that the exponent values depended on the exchange-interaction range characterized by $J(r)$ $=1 / r^{d+\sigma}$, where $d$ and $\sigma$ are the dimension and the interaction range, respectively, of the system. The meanfield-theory exponents are valid for $\sigma<\frac{1}{2}$ while the Heisenberg ones are valid for $\sigma>2$. The exponents belong to other universality classes if $\frac{1}{2}<\sigma<2$. Additionally, depending on the nature and structure of alloy ribbons (i.e., amorphous and/or nanocrystals embedded in an amorphous host) [10,21,22], long- and short-range magnetic interactions may coexist.

\section{CONCLUSION}

The magnetic and critical properties of the amorphous alloy ribbons, FCSNB-Ag and FCSNB-Cu, were studied in detail by means of $M(H, T)$ data. Experimental results revealed that FM-PM phase transitions take place in FCSNB-Ag and FCSNB-Cu at $T_{C} \approx 308 \mathrm{~K}$ and 322 $\mathrm{K}$, respectively. Using the SOMT theory, MAP method and scaling hypothesis, we determined the values of the critical exponents for the alloy ribbons, with $\beta=0.369$ $-0.376, \gamma=1.315-1.359$ and $\delta=4.5-4.7$. These values appear to be quite close to those expected for the $3 \mathrm{D}$ Heisenberg $(\beta=0.365$ and $\gamma=1.336)$ and reveal the presence of short-range magnetic interactions in the amorphous alloy ribbons. Because of the different values of magnetization and critical parameters $\left(T_{C}, \beta, \gamma\right.$ and $\delta$ ) obtained for two samples, we believe that the characteristic of the FM and PM phases in FCSNB-Ag and FCSNB-Cu depend not only on Cr-doping content but also on the physical nature of the elements $\mathrm{Ag}$ and $\mathrm{Cu}$ present in the FCSNB host.

\section{ACKNOWLEDGMENTS}

This research was supported by the National Foundation for Science and Technology Development (NAFOSTED) of Vietnam (103.02-2010.38). 


\section{REFERENCES}

[1] I. Betancourt, Materials 4, 37 (2011).

[2] H. G. Kim, M. S. Kim and W. N. Myung, J. Korean Phys. Soc. 49, 1630 (2206).

[3] T. D. Thanh, Y. K. Yu, P. T. Thanh, N. H. Yen, N. H. Dan, T. L. Phan, A. M. Grishin and S. C. Yu, J. Appl. Phys. 113, 213908 (2013).

[4] T. L. Phan, P. Zhang, N. H. Dan, N. H. Yen, P. T. Thanh, T. D. Thanh, M. H. Phan and S. C. Yu, Appl. Phys. Lett. 101, 212403 (2012).

[5] M. Naka, K. Hashimoto and T. Masumoto, J. Non-cryst. Solids 31, 355 (1979).

[6] Y. Yoshizawa, S. Oguma and K. Yamauchi, J. Appl. Phys. 64, 6044 (1998).

[7] T. Choh, H. Chihara, M. Igarashi, O. Kohmoto and Y. Narumiya, IEEE Translat. J. Magn. Jpn. 7, 384 (1992).

[8] A. Kolano-Burian, M. Kowalczyk, R. Kolano, R. Szymczak, H. Szymczak and M. Polak, J. Alloys Compd. 479, 71 (2009).

[9] C. X. Huu, N. Chau, N. D. The and N. Q. Hoa, J. Korean Phys. Soc. 53, 763 (2008).

[10] T. D. Thanh, N. H. Yen, P. T. Thanh, N. H. Dan, P. Zhang, T. L. Phan and S. C. Yu, J. Appl. Phys. 113, 17E123 (2013).

[11] V. Franco, J. S. Blazquez, B. Ingale and A. Conde, Annu. Rev. Mater. Res. 42, 305 (2012).

[12] S. G. Min, L. G. Ligay, K. S. Kima, S. C. Yu, N. D. Tho and N. Chau, J. Magn. Magn. Mater. 300, e385 (2006).

[13] C. F. Conde, M. Millan, J. M. Borrego, A. Conde, M. J. Capitan and J. L. Joulaud, Philosophical Magazine Lett. 78, 221 (1998).

[14] J. M. D. Coey, Magnetism and Magnetic Materials (Cambridge University Press, Cambridge, 2010).

[15] H. E. Stanley, Introduction to Phase Transitions and Critical Phenomena (Oxford University Press, London, 1971).

[16] A. Arrott, Phys. Rev. 108, 1394 (1957).

[17] A. Arrott and J. E. Noakes, Phys. Rev. Lett. 19, 786 (1967).

[18] B. M. Wang, L. Wang, Y. Liu and B. C. Zhao, J. Appl. Phys. 105, 023913 (2009).

[19] I. M. Kyprianidis, C. A. Achilleos, I. A. Tsoukalas, H. Bremers and J. Hesse, J. Magn. Magn. Mater. 161, 203 (1996).

[20] A. Perumal, V. Srinivas, K. S. Kim, S. C. Yu, V. V. Rao and R. A. Dunlap, Phys. Rev. B 65, 064428 (2002).

[21] F. J. Castano, J. M. Garcia-Beneytez, P. Crespo, M. Multigner, M. Vazquez and A. Hernando, J. Phys. Condens. Matter 11, 5671 (1999).

[22] V. Franco, R. Caballero-Flores, A. Conde, K. E. Knipling and M. A. Willard, J. Appl. Phys. 109, 07A905 (2011).

[23] R. Skomski, Simple Model of Magnetism (Oxford University Press, London, 2008).

[24] M. E. Fisher, S. K. Ma and B. G. Nickel, Phys. Rev. Lett. 29, 917 (1972). 